\title{
Chile: las jornadas electorales y la nueva democracia
}

Marcelo García Silva

1 15 de octubre de 1988 (plebiscito) y el 14 de diciembre de 1989 (elecciones presidenciales y parlamentarias) constituyen dosfechasunánimemente reconocidas como cruciales, históricas y decisivas en las luchas del pueblo de Chile por impedir la perpetuación del régimen militar y buscar la transición y consolidación de la democracia. En ambos procesos electorales el proyecto autoritario salió doblemente derrotado; primero, en la persona de quien encabezó el golpe de Estado y la dictadura durante estos 16 años; después en la figura de quien encarnó el modelo neoliberal que busca imponer, con relativos éxitos, un proyecto de modernización parcial a costos demasiado eleva- dos para la mayoría de los chilenos. Ambas jornadas electorales son la expresión de una nueva realidad nacional, a partir de la cual, los futuros obstáculos y dificultades de la transición y democratización de la sociedad chilena se darán bajo un escenario diferente: el que conquistaron las fuerzas políticas de centro e izquierda con el apoyo de la mayoría de los chilenos en las pasadas elecciones.

Un aspecto significativo de esta transición es el carácter tranquilo y pacífico del retorno a la democracia, no obstante indudables periodos de tensión. El 5 de octubre la ciudadanía se opuso a que Augusto Pinochet permaneciera en el poder hasta 1997 y el dictador reconoció 
su derrota, creândose así las condiciones para las elecciones presidenciales y parlamentarias. Lenta pero progresivamente el país comenzó la búsqueda de su reencuentro con las tradiciones políticas democráticas y repudió el proyecto dictatorial de suspensión del sistema de partidos.

Diversas circunstancias contribuyen a explicar el carâcter pacífico de esta transición. En primer lugar, la reconocida tradición democrática y legalista de la cultura política chilena. Pese a estos 16 años de represión, ella sobrevivió e hizo posible que un amplio espectro de fuerzas opositoras dejaran de lado sus diferencias y se unieran tras la búsqueda de un propósito común: el fin de la proyección en el tiempo del régimen militar. En segundo, la propia decisión de las fuerzas armadas, que se manifestaron dispuestas a respetar la fórmula de transición establecida por ellas mismas en su Constitución de 1980 oponiéndose a los intentos de desconocer la derrota de su comandante en jefe el 5 de octubre. En tercero, a la actual estabilidad económica que vive el Chile de hoy, con niveles de inflación relativamente controlados, aumento considerable de los productos de exportación, control del déficit y reducción del monto de la deuda externa. (Evidentemente se trata de un éxito económico relativo, pues se ha impuesto a costa de una reducción de los gastos sociales y una severa restricción de los salarios; sin embargo, en un país con una poderosa clase media, niveles relativamente bajos de extrema pobreza y rodeado de naciones con severos problemas políticos y económicos, esta situación contribuyó a reforzaruna imagen de estabilidad que pesó de manera significativa en la conformación de un escenario de transición con menos ciclos de tensión social y represión política del que pronosticaban algunos sectores al iniciarse la transición.)

No obstante esta perspectiva promisoria, el panorama general del retorno democrático se ve amenazado por numerosas dificultades que podrían comprometer la propia transición y consolidación democrática. El gobierno saliente de Pinochet ha intentado limitar el margen de acción del próximo, gobierno, dictando diversas "leyes de amarre" (ley orgánica de las fuerzas armadas, reforma a la ley del Banco Central; ley de televisión) que persiguen crear verdaderos "enclaves institucionales" autónomos del gobierno civil entrante. Además, las fuerzas armadas se oponen terminantemente a que se las enjuicie por abusos a los derechos humanos durante la fase más represiva del gobierno militar. Otro factor inquietante es el estado de desorganización en que se encuentra la derecha chilena. Si no se estructura en torno a un polo de aglutinación capaz de vivir la política como un enfrentamiento entre adversarios dentro de una democracia política, existe el peligro de que emerja nuevamente la tentación del recurso a los métodos no democráticos por parte de sus sectores extremos. También son inquietantes los aspectos excluyentes en que se basa el éxito económico chileno, pues al no existir el respaldo activo de las fuerzas armadas a dicho modelo, el nuevo gobierno tendrá que soportar la presión de las demandas sociales de sectores largamente excluidos, que verán en la nueva democracia una oportunidad para mejorar sus niveles de vida. Por último, existe el peligro del resquebrajamiento de la alianza mayoritaria de centro e izquierda, cuando una vez superadas las circunstancias que los llevaron a suspender sus diferencias, renazcan las tendencias a competir por el liderazgo y el poder electoral poniendo 
en peligro la estabilidad del conjunto de la alianza mayoritaria.

Pese a la seriedad de estas dificultades, existen sin embargo motivos para mantener razonables dosis de optimismo ante la democracia que se inaugura. En efecto: en el proceso transcurrido entre las dos jornadas electorales, ninguna de las fuerzas representativas de las distintas culturas políticas se lograron plasmar, por encima de las demás, una visión exclusiva del cambio y la democracia. La necesidad de tener que ceder en una parte de sus respectivos ideologismos y utopías en beneficio de un crecimiento gradual de la democratización ha hecho posible un proceso de negociación y consensos que, de continuar, abrirá nuevas posibilidades para que en el Chile de hoy se instaure un régimen político más negociador, consensual y gradual, sin los niveles de polarización que llevaron al quiebre de la democracia en el pasado.

A continuación analizamos brevemente algunas condiciones políticas del periodo entre el 5 de octubre de 1988 y el 14 de diciembre de 1989; enseguida examinamos algunas tendencias de los últimos resultados electorales y finalmente exponemos las condiciones políticas en que se inaugura la democracia en Chile.

El plebiscito del 5 de octubre de 1988 tuvo múltiples connotaciones y planteó un conjunto de condicionantes en el periodo previo a las elecciones presidenciales y parlamentarias. Por una parte significó el fin de la pretensión de continuar la dictadura a través de un régimen autoritario como el previsto por la Constitución del 80. También significó el fin del proyecto de Pinochet de mantenerse en el poder para asegurar el paso del

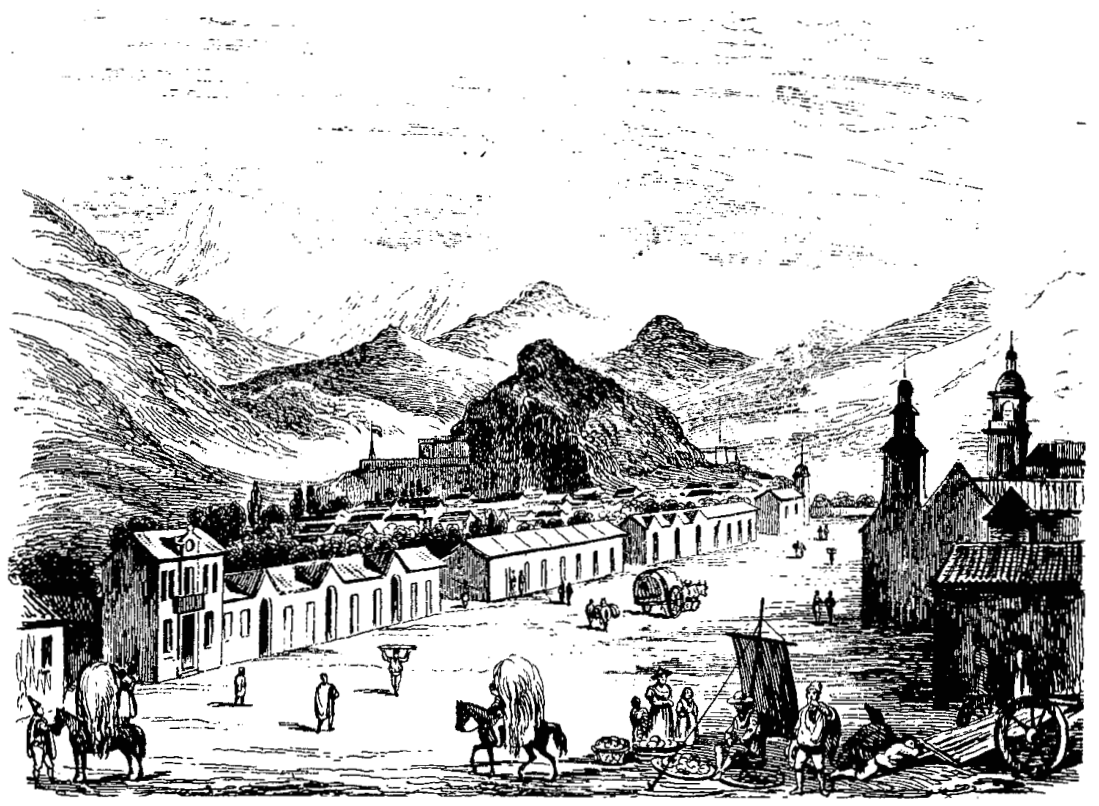


régimen de dictadura militar a régimen autoritario. Por otra parte, desencadenó un proceso de transición a la democracia que se desarrollaría dentro de plazos y mecanismos establecidos por el régimen, pero que resultarían modificadosen parte por la oposición.

Para Pinochet, significó el fin de la ilusión del dictador de controlar hasta su muerte los destinos del país. Para las fuerzas armadas, la derrota de su proyecto de liberar al régimen político chileno del sistema de partidos reemplazándolo por uno nuevo que perpetuara el papel tutelar de las fuerzas armadas y asegurar un tipo de transición de militares a civiles donde se garantizara un sistema de "democracia protegida" con relaciones verticales entre los individuos, las organizaciones intermedias y el Estado.

Indudablemente el régimen militar tuvo éxito en implantar una serie de condicionantes de tipo institucional, actoral y ético-simbólico que hoy funcionan como importantes "enclaves" a ser removidos por la nueva democracia. Donde falló sin embargo fue en no considerar que el modelo de modernización excluyente provocaría grandes dosis de frustración y resentimientos en los sectores medios y bajos, al pretender imponer mecánicamente un modelo en beneficio de pocos al costo del sacrificio de muchos, y al no tomar en cuenta que, enuna sociedad comola chilena, defuertes tradiciones democráticas y diferentes culturas y subculturas políticas, no era tan fácil acabar con el sistema de partidos no obstante la fuerza y la persistencia de los cambios económicos y sociales.

Para la oposición, el desencadenamiento de un proceso de transición a partir del 5 de octubre tuvo varios significados. Primero, la convirtió, por primera vez en estos quince años, en el actor principal y dirigente del proceso político.
Segundo, significó el triunfo dela estrategia de buscar el retorno pacífico a la democracia enfrentando a la dictadura en su propia legalidad. Tercero, enfrentó a la oposición con la tarea de llegar a las elecciones presidenciales y parlamentarias en las mejores condiciones para asegurar un gobierno mayoritario y de transición a la democracia. La evidencia de esta tarea en los medios de la oposición favoreció el que perdieran importancia otras tendencias y se impusiera finalmente la línea a favor de constituir una mayoría sociopolítica de centro e izquierda que enfrentara con un solo candidato presidencial, un programa común de gobierno y un pacto electoral parlamentario las primeras elecciones de diciembre de 1989.

Para la derecha, el plebiscito tuvo también una triple significación: por un lado, la derrota del régimen la tomó poco preparada para competir democráticamente, después de vivir quince años a la sombra de una dictadura que, por tener una desconfianza profunda en la política y una fe ciega en la disciplina y la autoridad, había hecho muy poco por revitalizar a los partidos de derecha. Por otro, su dependencia del régimen no favoreció el que la derecha pudiera imponer una línea de acción más independiente del gobierno levantando una coalición másabierta hacia la centro derecha, como lo proponía en un comienzo el partido Renovación Nacional, sino que prevaleció la tendencia de los sectores puristas más comprometidos con el modelo económico de la dictadura que guardaban algunas suspicacias respecto a la veracidad del pleno compromiso de Renovación Nacional con las políticas económicas del régimen militar; fue por esto que decidieron enfrentar las elecciones presidenciales con una figura más" "genuina" del experimento económico neoli- 
beral como la del ex ministro de hacienda de Pinochet, Hernán Buchi. Por último, la derecha no logró, sin embargo enfrentar las primeras elecciones con un solo candidato, pues, si bien Renovación Nacional aceptó de mala gana retirar la candidatura de Jarpa y a poyar la de Buchi, un sector perseveró en el intento de provocar una segunda vuelta levantando una dictadura populista de derecha que fuera capaz de obtener los votos de los descontentos con la gestión económica del gobierno militar.

En el periodo entre las dos jornadas electorales se desarrollaron otros procesos sumamente importantes de tener en cuenta para entender correctamente las condiciones bajo las cuales se inaugura la democracia en Chile. Uno fue el delicado proceso de reformas constitucionales antes de la elección. Otro, el mecanismo que escogió el gobierno para elegir a los candidatos al Congreso.

La oposición democrática trató durante este periodo de eliminar los aspectos más autoritarios de la Constitución del 80: la prohibición de los partidos marxistas, el nombramiento por el gobierno de aproximadamente un tercio del Senado, las facultades de veto del Consejo de Seguridad Nacional sobre las autoridades civiles y los difíciles requisitos que se tenían que cumplir para modificar la Constitución. Después de un extenuante proceso de negociación, el paquete de reformas que aprobó la ciudadanía en el referéndum del 30 de julio recogió muchas de las preocupaciones de la oposición: la interdicción de los partidos marxistas se redujo a una prohibición de los grupos que practicaban la violencia política; se incrementó el numero de senadores elegidos para reducir el peso de los senadores designados; se redujo el poder del Consejo de Seguridad Nacional; se incorporaron al- gunas disposiciones que reforzaran la protección a los derechos humanos y se introdujeron cambios sustanciales a fin de facilitar las reformas a la Constitución por el próximo gobierno.

Si en las negociaciones sobre las reformas constitucionales el régimen aceptó hacer algunas concesiones políticas, en la elaboración de las leyes electorales en cambio optó por un sistema que le diera las mayores ventajas a sus candidatos en la competencia por el Congreso. Así, la Junta elaboró primero un conjunto de disposiciones para dividir arbitrariamente los distritos electorales de manera que a las áreas rurales, donde el voto progubernamental había sido más fuerte en el plebiscito, les correspondería un mayor número de diputados que a las áreas urbanas donde el voto contra la dictadura había sido superior. Enseguida la Junta opto por una mecánica binominal de ley electoral que premiaba en definitiva a la primera minoría. De acuerdo con este sistema, la ley electoral dispuso que en cada distrito electoral habria dos cargos a ser llenados por el proceso electoral. Los votantes elegirían un candidato entre las respectivas listas que presentaran los partidos y los ganadores serian seleccionados de acuerdo con el total de votos recibidos por la lista. La que recibiera el mayor número de votos ganaría un cargo al Congreso y la siguiente lista que obtuviera a lomenos la mitad de esos votos ganaría el segundo, de manera que si se presentaban dos listas, la primera podía obtener hasta $65 \%$ de los votos y todavía sacar un solo cargo; mientras la segunda, con sólo $33 \%$ de las preferencias, obtener el segundo.

El gobierno jugaba además con la posibilidad de que los 17 partidos de la oposición no llegarian a conformar una sola lista. Sin embargo, contrariamente a lo esperado, los partidos de oposición nio 
sólo hicieron a un lado sus diferencias ideológicas y se pusieron de acuerdo para postular a Patricio Aylwin como candidato único de oposición a la presidencia, sino que convinieron en un programa de gobiemo y hasta en un pacto electoral que significó en la práctica una sola lista de candidatos de oposición al Congreso. Para que esto se produjera fue necesario un largo, complicado y a veces extenuante proceso de concertación dentro de cada partido de oposición y entre los partidos de la concertación entre sí, a fin de ponerse de acuerdo en el número de candidatos por partido y el lugar donde ellos se postularían. Finalmente sellegó a una sola lista nacional de oposición a la que se agregaron algunas listas regionales que incluyeron candidatos de fuera de la coalición aylwinista, como los del Partido Comunista que decidió finalmente apoyar a Aylwin y presentar candidatos al Congreso.

Las elecciones del 14 de diciembre fueron las primeras elecciones presidenciales en 19 años y las primeras de tipo parlamentario en 16 . Sus resultados arrojan un conjunto de magnitudes y tendencias que conviene analizar a fin de poder contar con una imagen más realista de su significado.

En lo que concierne a la elección presidencial, el resultado oficial final arrojó un claro triunfo del candidato de oposición sobre sus contendientes, alcanzando la cifra de $55.2 \%$ (Buchi $29.4 \%$ y Errazuriz $15.4 \%$ ). Esta cifra es muy similar a la que obtuvo la oposición No el 5 de octubre de 1988 que llegó a 56 porciento.

El NO obtuvo 3967579 votos y Patricio Aylwin sumó 3849584 sufragios. Por tanto, las elecciones recientes ratificaron los resultados del plebiscito y no un cambio fundamental en la correlación de fuerzas ni en la legitimidad que a nivel presidencial se requiere para encarar las tareas del primer gobierno democrático dentro de un régimen de transición a la democracia. Se observa igualmente que el intento derechista de provocar uná segunda vuelta levantando una candidatura populista que fuera capaz de obtener los votos de los descontentos con la gestión económica del gobierno militar, como la sugerida por la candidatura de Francisco Javier Errazuriz, no dio los resultados esperados.

Sin embargo, por debajo de esta similitud, existe una importante variación entre el voto No y el que se volcó por Patricio Aylwin cuando se observa lo ocurrido en las grandes comunas urbanas y las de tipo rural. Como se dijo anteriormente, el voto No fue más fuerte en las zonas urbanas que en las rurales. Sin embargo, en las elecciones recientes se constató una disminución del voto por Aylwin respecto al voto No en las grandes ciudades $(-4.6$ en Antofagasta, $-4.0 \%$ en Concépción, $-3.1 \%$ en Santiago, $-6 \%$ en Valdivia y $-6.6 \%$ en Valparaíso). A la inversa, la votación de Patricio Aylwin registró un aumento en las comunas rurales en relación con la votación por el NO en esas zonas. El fortalecimiento del voto opositor en las zonas rurales lo explican algunos por el fenómeno de la pérdida del temor que ya había ocurrido de alguna manera en las grandes ciudades en la campaña por el plebiscito. Quedan por explicar las razones de la caída del voto opositor en las áreas urbanas.

Si se observa la votación presidencial por regiones (trece regiones) se constatan tendencias que confirman la observación anterior. En regiones como la primera, segunda, quinta y metropolitana, de un claro contenido urbano, cae la votación opositora. Por el contrario, en las regiones décima y undécima, donde el voto sí predominó sin contrastes, la 
elección reciente le dio a Patricio Aylwin la mayoría absoluta.

En relación con las elecciones parlamentarias, en el Senado, de un total de 38 asientos en disputa, la oposición obtuvo 22 escaños, y en la Cámara de Diputados, de un total de 120 , consiguió 71 contra 49. Si se observan las elecciones para el Senado se pueden hacer las siguientes constataciones en cuanto a magnitud y tendencias por corrientes políticas nacionales.

En cuanto a magnitud, se destacan dostendencias de fondo. La primera que, en todas las regiones, la suma de la votación de la lista de la Concertación por la Democracia más la del pacto Partido Amplio de Izquierda (PAIs), Partido Radical Social Demócrata (en las regiones en que esta lista existió) arrojó un resultado porcentual más favorable aún para la oposición que el de la propia elección presidencial (59\% contra 55\%). La segunda, que la oposición resultó vencedora en todas las regiones del país con la sola excepción de la novena región donde tuvo apenas un 50\% (cuadro 1).

En relación con las tendencias por corrientes políticas, la izquierda obtuvo $23 \%$ de los votos, pero eligió solo a seis senadores de un total de 38 escaños en disputa, lo que representa tan sólo $15.8 \%$ de los cargos senatoriales de elección popular. Los partidos del centro político obtuvieron $36 \%$ de los votos y ganaron $42 \%$ de los escaños en disputa (trece los demócrata-cristianos y tres de los radicales). Mientras que el pacto derechista representado por la alianza electoral de Renovación Nacional (RN) con la Unión Democrática Independiente (UDI), con apenas un poco más del tercio de los votos eligió a 16 senadores, lo que representa aproximadamente $42 \%$ de los asien-

Cuadro 1

RESULTADOS DE LA ELECCIÓN PRESIDENCIAL 1989

$\begin{array}{lrrrrr}\text { Regiones } & \text { \% No } & \text { Aylwin } & \text { Buchi } & \text { Erraz } & \begin{array}{r}\text { Senadores } \\ \text { oposición }\end{array} \\ \text { I. Tarapaca } & \text { (a) } & (b) & & & \\ \text { II. Antofagasta } & 55.3 & 49.7 & 31.5 & 18.8 & 63.9 \\ \text { III. Atacama } & 60.7 & 57.6 & 24.8 & 17.5 & 65.0 \\ \text { IV. Coquimbo } & 56.2 & 60.7 & 30.3 & 9.0 & 61.7 \\ \text { V. Valparaíso } & 54.0 & 57.2 & 31.0 & 12.2 & 60.3 \\ \text { VI. B. O'Higgins } & 57.3 & 52.6 & 29.0 & 18.4 & 57.6 \\ \text { VII. del Maulle } & 51.9 & 58.7 & 29.6 & 11.7 & 59.2 \\ \text { VIII. Biobio } & 55.3 & 57.0 & 28.5 & 14.5 & 59.9 \\ \text { X. Araucania } & 45.9 & 56.3 & 24.9 & 18.8 & 62.5 \\ \text { X. Los Lagos } & 49.8 & 57.2 & 28.6 & 24.2 & 50.0 \\ \text { XI. Ibáñez del C. } & 50.0 & 54.1 & 29.0 & 19.9 & 57.0 \\ \text { XII. Magallanes } & 57.6 & 60.4 & 30.9 & 14.4 & 57.3 \\ \text { Reg. Metropolitana, } & & & 29.6 & 10.1 & 66.2 \\ \text { Santiago } & 59.0 & 56.2 & 31.3 & 12.5 & 59.7 \\ & & & & & \\ \text { Total } & 56.0 & 55.2 & 29.4 & 15.4 & 59.0\end{array}$

Fuente: Tribunal Calificador de Elecciones 
Cuadro 2.

RESULTADOS DE SENADORES 1989

$\begin{array}{lccc} & \begin{array}{c}\text { Votos por } \\ \text { partido }\end{array} & \begin{array}{c}\% \\ \text { País }\end{array} & \begin{array}{c}\text { Senadores } \\ \text { electos }\end{array} \\ \text { PPD } & & & \\ \text { PS (Arrate) } & 799359 & 12.0 & 10.0 \\ \text { PS(Almeyda) } & 687694 & 10.3 & 7.9 \\ \text { PC } & 268464 & 4.0 & 2.6 \\ \text { PRSD } & 163561 & 2.5 & - \\ \text { IC } & 136791 & 2.1 & 2.6 \\ \text { PH } & 124792 & 1.9 & - \\ \text { Total } & 35643 & 0.5 & - \\ \text { izquierda } & 1528610 & 23.0 & 15.8 \\ \text { PDC } & & 32.2 & 34.2 \\ \text { PR } & 2141012 & 2.2 & 5.3 \\ \text { SD } & 146062 & 0.7 & 2.6 \\ \text { Independiente } & 40658 & 0.9 & - \\ \text { Total centro } & 59152 & 36.0 & 42.1 \\ & 2394884 & 10.9 & \\ \text { RN } & & 5.1 & 13.2 \\ \text { UDI } & 723250 & 19.1 & 5.3 \\ \text { Independiente } & 338043 & & 23.7 \\ \text { Total uDI-RN- } & 1272594 & 35.1 & 42.1 \\ \text { Independiente } & 2333892 & & \end{array}$

Fuente: Servicio electoral, Gonzalo D. Martner, Convergencia, núm 17, enero-marzo de 1990, p. 10.

tos senatoriales. Si a éstos se suman los nueve senadores designados por el gobierno, resulta que la oposición, con $59 \%$ de los votos, terminó teniendo $47 \%$ de los asientos del Senado. La penalización fue aun mayor dentro de las filas de la izquierda, donde hubo candidaturas como las de Ricardo Lagos y Luis Maira que no obstante alcanzarla segunda mayoría en cada una de sus respectivas regiones, no resultaron elegidas y sí lo fueron contendientes con mucha menor votación. Ricardo Lagos por ejemplo, con la tercera mayoría individual nacional no resultó elegido; en cambio sí resultó ganador su contendiente que obtuvo
160000 votos menos en una lista con 360000 menos que la de la oposición. En consecuencia, pese a que el voto popular favoreció a la oposición por amplia mayoría en la Cámara de Diputados y por una mayoría mas estrecha en el Senado, al final de cuentas, la mecánica de nominación binominal ideada por el gov bierno militar permitió premiar a la primera minoría que en este caso resultó ser la derecha del pacto UDI-RN. En síntesis, la oposición nocuenta por sí misma conla mayoría necesaria para integrar los quorum de parlamentarios necesarios para introducir las modificaciones institucionales fundamentales que permitan 


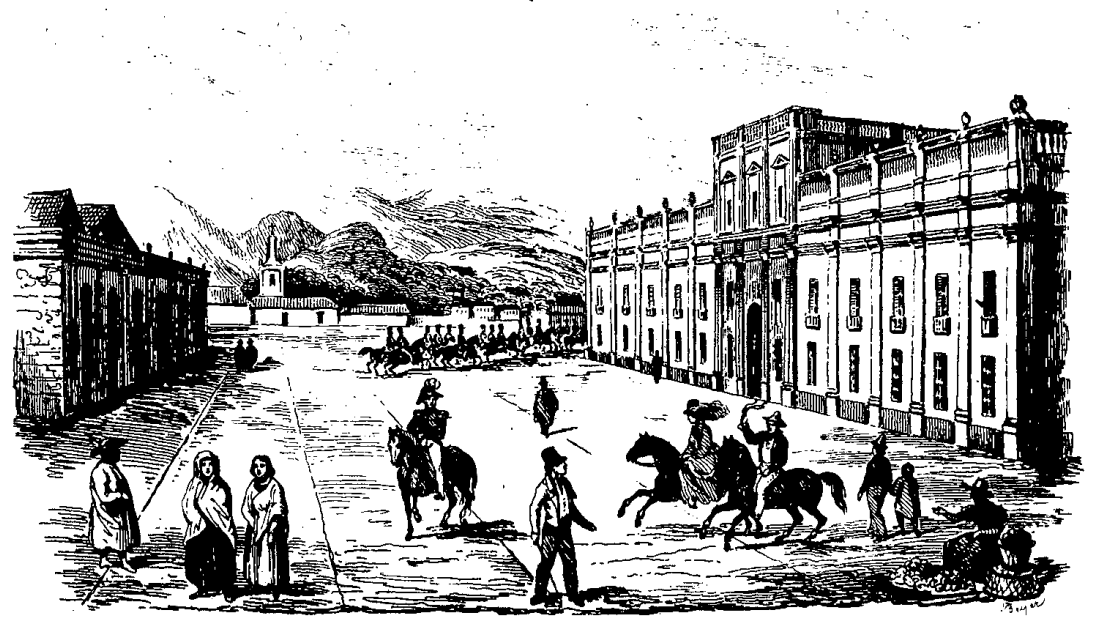

completar el proceso de transición, por lo que deberá acudir a nuevos acuerdos restrictivos con la derecha política para continuar las reformas constitucionales.

¿Cuáles son las condiciones y dificultades con las que se inaugura la democracia en Chile y qué probabilidades tiene el nuevo gobierno de asegurar la consolidación de esa democracia con cambio social?

En general, esas condiciones tienen que ver, por una parte, con las situaciones quela transición hereda del pasado y que se conocen como los "enclaves autoritarios". Con ello hacemos referencia a los rasgos de tipo sociocultural dejados por la dictadura: los amarres institucionales y las relaciones con las fuerzas armadas y los derechos humanos. Porotra, con los problemas de la modernización económica y las condiciones para la constitución y desarrollo del primer gobierno democrático.
En relación con los rasgos socioculturales, es indudable que la democracia que se inaugura se encuentra con una sociedad que ha vivido por más de quince años bajo un régimen que a través de cambios estructurales, constitucionales y uso de la represión buscó imponer un modelo nuevo de relaciones entre Estado y sociedad, lo mismo que cambiar actitudes y mentalidades en los sujetos y actores sociales: fomento del individualismo y de la cultura empresarial, erradicación de la política, elitización y fragmentación de la sociedad, etc. Sin embargo, si bien todos estos rasgos son ciertos, también lo es que en la sociedad chilena no se ha producido el tipo de anomia, conformismo o alienación propias de este tipo de dictaduras. Por el contrario, las movilizaciones sociales a partir de 1983, la reconstitución de organizaciones sociales y la mayor politización de la'sociedad provocada por el 


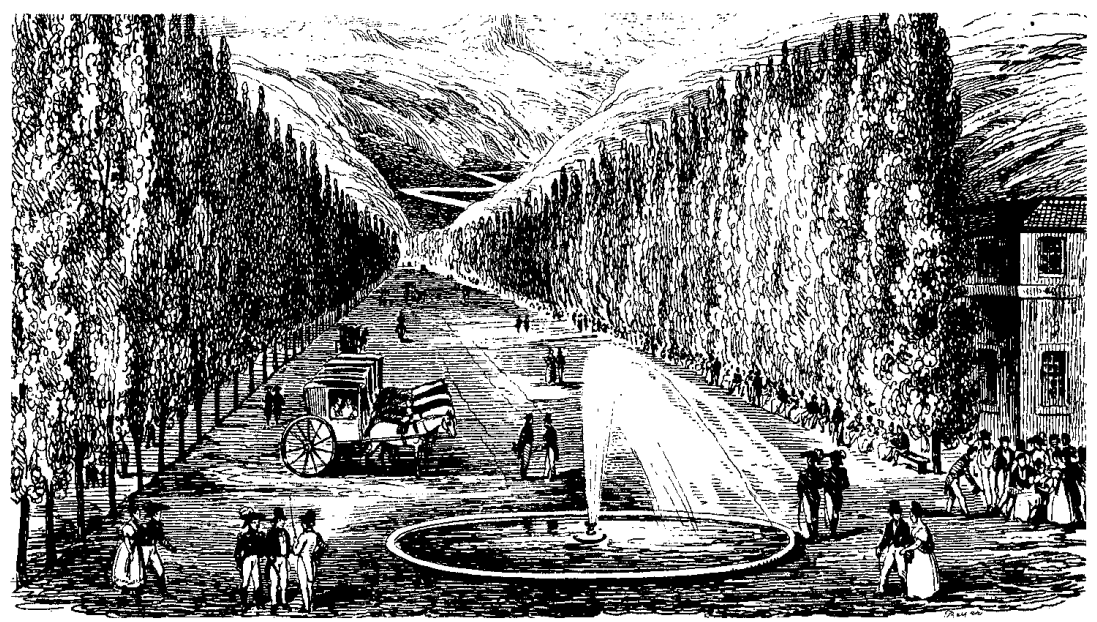

proceso mismo de transición (plebiscito, inscripción de partidos, jornadas electorales, etc.) permiten sugerir que, en el Chile de hoy, todavía se mantiene una tradición cultural democrática que es la que permite a la gente participar, reconocer y reubicarse dentro del espectro de partidos.

Respecto de los llamados "enclaves institucionales", es decir las reformas constituciónales que quedaron pendientes y que todavía marcan un rasgo autoritario, lo mismo que otras cuestiones más específicas (leyes sobre el Banco Central, las fuerzas armadas, el Congreso, la educación, las leyes laborales, etc.). Aquí hay que tomar en cuenta dos aspectos: por un lado, que la superación de estos enclaves va a requerir de acuerdos y mayorías políticas que vayan mas allá de las miras de la actual coalición y que esto se va a traducir en tiempo y concesiones mayores que podrían repercutir tanto en la cohesión de la actual coalición como en la erosión de su fuerza y credibilidad frente a la opinión pública. Por el otro, que la alta legitimidad alcanzada por el presidente y la concertación pueden ser aprovechadas durante el primer tiempo democrático para la movilización de actores en ámbitos específicos y para que algunos sectores de la derecha y del empresariado traten ellos también de no perder legitimidad apoyando los avances institucionales.

En relación con las fuerzas armadas, el nuevo gobierno enfrenta numerosas dificultades: las prerrogativas del general Pinochet en el mando del ejército, la excesiva autonomía y franquicias político-corporativas de las fuerzas armadas y el rechazo rotundo del ejército a la cuestión de los juicios y castigos por las violaciones a los derechos humanos. Aquí la solución exitosa que le dé el gobierno a estos problemas va a depender del cambio de la correlación de fuerzas y de la legitimidad del gobierno frente a la población. Cambiando esa correlación va a ser posible reducir la influencia política del ejército. Dependiendo de esa correlación de fuerzas y legitimidad, el gobierno podrá negociar la autonomía y prerrogativas del ejército a cambio de no 
tomar porsi mismola iniciativa de someter a juicio a las fuerzas armadas, sin perjuicio de que favorezca mediante reformas legales el que, frentè a determinadas denuncias concretas de personas afectadas, los tribunales ordinarios cumplan sus funciones. Sin embargo, en ningún caso hay que descartar la posibilidad de que esto plantee dificultades en el interior de una coalición gobernante que ha sido afectada de manera muy diferente por los crímenes de los militares.

Por último están las condiciones relacionadas con el modelo económico y las relaciones entre política y sociedad. Aquí no hay que perder de vista la posibilidad de la llamada "explosión de demandas reprimidas" que en un inicio pudieran desestabilizar la economía o crear problemas de gobernabilidad. Sin embargo hay que tener en cuenta tres aspectos que le restan potencial explosivo a este problema: a) Chile, a diferencia de otros procesos de transición, no enfrenta una crisis económica aguda que obligue al nuevo gobierno a imponer planes de emergencia que aumenten las tensiones de la sociedad ni enfrenta tampoco presiones desmedidas de la sociedad por demandas insatisfechas; $b$ ) de acuerdo con algunos estudios realizados, la mayoría de las demandas muestran un bajo grado de expectativas en cuanto a que es posible esperar durante el primer tiempo del gobierno democrático. La mayoría tienen que ver con salud, educación, vivienda y mayor participación, todas ellas compatibles con el plan que tiene el actual gobierno de continuar con el modelo económico de economía abierta y competitivo pero introduciéndole reformas para mejorar la situación de los mas desfavorecidos; $c$ ) en Chile la constitución delas demandassocialesha pasado siempre por los actores partidarios, por lo que el fenómeno del espontaneísmo explosivo de las demandas no se da con la violencia de otras partes, y $d$ ) hoy participan en la coalición gobernante la mayoría de los partidos que podrían activar esas demandas, de manera que es posible pensar que el programa económico del nuevo gobierno cuente con mayores grados de legitimidad.

Las condiciones y tareas mencionadas remiten finalmente al problema de la constitución y estabilidad del primer gobierno de transición. Esto se refiere, por una parte, al tipo de mayoría social y política que requiere el futuro gobierno, y por otra al modo de funcionamiento de dicha mayoría. El primer aspecto Chile lo ha resuelto conformando una gran alianza del centro con la izquierda donde lo que busca es superar el gran problema de la ruptura entre las clases medias y los sectores populares. Problema en buena medida responsable del derrumbe democrático y de la imposibilidad para asegurar una democracia política con cambio social.

El segundo aspecto, aquél de cuál deba ser el modelo más adecuado que garantice el mejor funcionamiento de la coalición gobernante está todavía por dilucidarse. Aquí lo que está en juego es cómo asegurar la competencia interna de la coalición sin romperla y de qué manera garantizar la diferenciación necesaria para competir por el liderazgo sin destruir la fuerza del propio gobierno.

Aquí la tendencia que parece imponerse para el próximo horizonte histórico es la de una alianza entre el centro y la izquierda, entre la Democracia Cristiana y el socialismo chileno como las dos fuerzas preponderantes que aspiran al liderazgo hegemónico de la coalición gobernante, pero enuna especie de "pacto de alternancia regulada" que no rompa la mayoría social y política de la actual coalición. 


\section{SECUENCIA}

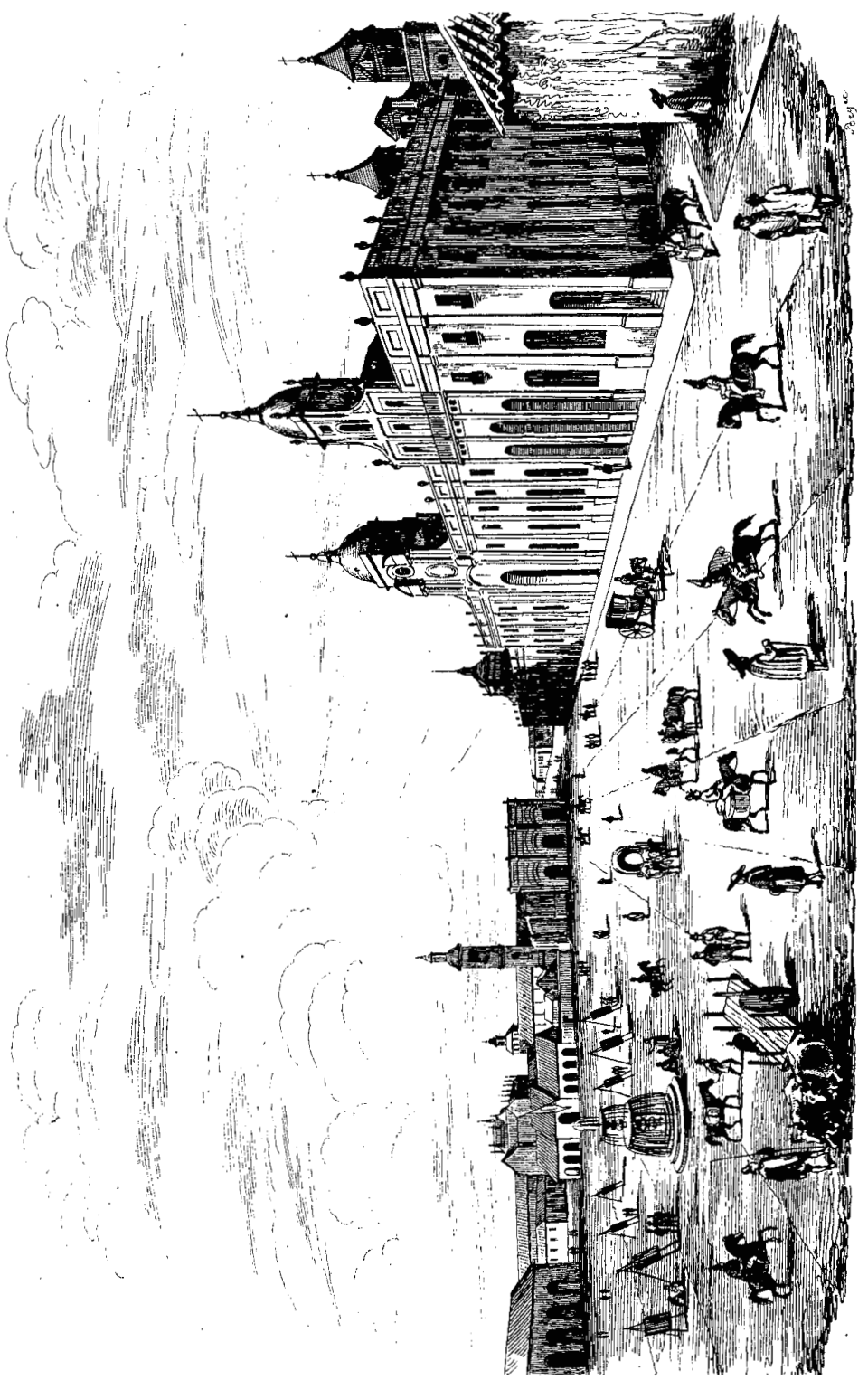

